\title{
Comunidade ictiofaunística do córrego Moeda, bacia do Alto Paraná: composição, estrutura e atributos ecológicos
}

Ichthyofaunistic community of the Moeda Stream, Upper Paraná basin: composition, structure and ecological attributes

Comunidad ictiofaunística del arroyo Moeda, cuenca del Alto Paraná: composición, estructura y atributos ecológicos

Letícia de Oliveira Manoel Doutoranda em Ciências Biológicas, UNESP/IBB, Brasil. leticia.is@gmail.com

Nereida Vilalba Alvares de Almeida Mestre em Geografia, UFMS, Brasil. almeidanereida2@gmail.com

Maria José Alencar Vilela Professora Doutora, UFMS, Brasil. mjavilela@yahoo.com.br 


\begin{abstract}
RESUMO
Objetivou-se neste estudo avaliar a estrutura e a composição da ictiofauna do córrego Moeda, afluente da margem direita do rio Paraná, município de Três Lagoas, MS. Foram efetuadas oito coletas trimestrais entre fevereiro/2012 e novembro/2013, em duas áreas amostrais: P1, no médio curso e P2, na foz do córrego Moeda. A riqueza de espécies, a abundância numérica e os índices de diversidade Shannon-Wiener e de equitabilidade de Pielou foram estimados para cada sítio amostral. Para verificar diferenças na estrutura da comunidade entre as áreas aplicou-se a análise PERMANOVA e, posteriormente, SIMPER. Foram capturados 296 exemplares, distribuídos em cinco ordens, 17 famílias, 33 gêneros e 41 espécies. Não se observou diferença quanto à abundância numérica e à equitabilidade entre as áreas avaliadas. Contudo, houve diferença quanto à riqueza de espécies e à diversidade. Tal resultado pode estar relacionado à maior heterogeneidade de habitat em P2, que oferecem locais para forrageamento, reprodução e abrigo, além de maior proximidade à calha do rio Paraná. No P1, ao contrário, a mata ripária encontra-se reduzida, ausente em alguns trechos, refletindo em maior assoreamento, menor profundidade e menor variabilidade do substrato, aspectos que devem colaborar para a diminuição da diversidade de peixes. Também observamos diferenças na estrutura da comunidade, sendo Knodus moenkhausii e Astyanax lacustris as espécies que mais contribuíram para a diferença. Os resultados destacam a importância da preservação da vegetação marginal dos riachos, essencial à manutenção da biota aquática.
\end{abstract}

PALAVRAS-CHAVE: Biodiversidade. Peixes. Conservação ambiental.

\begin{abstract}
In this study we evaluated the structure and composition of the ichthyofauna of the Moeda Stream, a tributary of the Paraná River, in the municipality of Três Lagoas, MS. Eight quarterly collections were made between February/2012 and November/2013, in two sampling sites: P1, middle course, and P2, at the mouth of the Moeda Stream. Species richness, numerical abundance and Shannon-Wiener diversity and Pielou equitability indexes were estimated for each area. In order to verify differences in the structure of the community between the areas we applied a PERMANOVA analysis and SIMPER. A total of 296 specimens were collected, distributed in five orders, 17 families, 33 genera and 41 species. No difference was observed in the numerical abundance and the equitability between the areas. However, there was a difference in species richness and diversity. This result may be related to the higher habitat heterogeneity in P2, which offer sites for foraging, breeding and shelter, as well as closer proximity to the Paraná river channel. In $\mathrm{P} 1$, in contrast, the riparian forest is reduced or even absent, reflecting in higher sedimentation, lower depth and lower substrate variability, aspects that should contribute to the decrease of fish diversity. We also observed differences in the structure of the community, with Knodus moenkhausii and Astyanax lacustris being the species that contributed most to the difference. The results highlight the importance of preserving the marginal vegetation of the streams, essential for the maintenance of the aquatic biota.
\end{abstract}

KEYWORDS: Biodiversity. Fishes. Environmental Conservation.

\title{
RESUMEN
}

Se objetivó en este estudio evaluar la estructura y composición de la ictiofauna del arroyo Moeda, afluente del río Paraná, municipio de Três Lagoas, MS. Efectuamos ocho colectas trimestrales entre febrero/2012 y noviembre/2013, en dos sitios: P1, en el medio curso y P2, en la desembocadura del arroyo. La riqueza de especies, abundancia numérica y índices de diversidad Shannon-Wiener y equidad de Pielou fueron estimados para cada sitio. Para verificar diferencias en la estructura de la comunidad entre las áreas se aplicó el análisis PERMANOVA y SIMPER. Se capturaron 296 ejemplares, distribuidos en cinco órdenes, 17 familias, 33 géneros y 41 especies. No se observó diferencia en cuanto a la abundancia numérica y a la equidad entre las áreas. Sin embargo, hubo diferencia en cuanto a la riqueza de especies y a la diversidad. Este resultado puede estar relacionado con la mayor heterogeneidad de hábitat en P2, que ofrece locales para forraje, reproducción y abrigo, además de mayor proximidad al canal del río Paraná. En el P1, por el contrario, la mata riparia se encuentra reducida, ausente en algunos tramos, reflejando en mayor sedimentación, menor profundidad y menor variabilidad del sustrato, aspectos que deben colaborar para la reducción de la diversidad de peces. También observamos diferencias en la estructura de la comunidad, siendo Knodus moenkhausii y Astyanax lacustris las especies que más contribuyeron a la diferencia. Los resultados destacan la importancia de la preservación de la vegetación marginal de los arroyos, esencial para el mantenimiento de la biota acuática.

PALABRAS CLAVE: Biodiversidad. Peces. Conservación ambiental. 


\section{INTRODUÇÃO}

A fauna íctica de água doce da América do Sul possui uma grande diversidade e complexidade; no entanto, o conhecimento da ecologia, biologia e sistemática desse grupo, apesar de crescente, mostra-se ainda incompleto (VARI \& MALABARBA, 1998). Estima-se que mais de 6.000 espécies de peixes de água doce sejam encontradas na região Neotropical, 4.475 das quais já reconhecidas e outras 1.550 ainda não descritas (REIS et al. 2003). Deste total, cerca de 2.500 habitam águas doces brasileiras, número que provavelmente é mais elevado, dada a grande quantidade de estudos em andamento que têm resultado em registros de novas espécies (GRAÇA e PAVANELLI, 2007).

Em território brasileiro a bacia do Alto Rio Paraná abrange mananciais dos estados de Goiás, Mato Grosso do Sul, Minas Gerais, Paraná e São Paulo e do Distrito Federal. Corresponde ao primeiro terço da bacia do rio Paraná, ocupando uma área de $891.000 \mathrm{~km}^{2}(10,5 \%$ do território brasileiro) (AGOSTINHO et al., 2004).

Essa bacia possui um grande número de espécies de peixes (310 descritas e 60 em diferentes fases de descrição), com expectativa de descoberta de novos táxons, frente ao crescimento de estudos em regiões pouco exploradas (LANGEANI et al., 2007). No entanto, sua integridade tem sido fortemente afetada pela crescente ação antrópica. A ictiofauna tem sido impactada por diversos fatores, dentre os quais cabe destacar a construção dos grandes reservatórios para produção de energia, poluição por diversas fontes, pesca predatória, emissão de efluentes domésticos e industriais, alterações ambientais na bacia, incluindo desmatamentos e práticas incorretas de uso de solos, além de atividades inadequadas de manejo da ictiofauna, como introdução de espécies não nativas (AGOSTINHO \& GOMES, 2006). Tais impactos resultam em alterações não só na ocorrência das espécies, mas também na composição relativa das comunidades.

Segundo Casatti et al. (2010) a antropização de áreas ripárias promove mudanças de habitats e alterações na disponibilidade de recursos alimentares para ictiofauna, caracterizando-se como uma grave ameaça à diversidade aquática. Sua ausência ou escassez acarreta aumento de sedimentos, maior luminosidade no curso do rio, podendo acarretar em diminuição de espécies intolerantes e aumento de espécies generalistas (LORION \& KENNEDY, 2009).

Nos riachos da região de Três Lagoas, além dos fatores já citados, a substituição das coberturas vegetais nativas por pastagens e, mais recentemente, por extensos plantios de eucalipto, também são potencialmente causadores de alterações ambientais que podem afetar as comunidades desses sistemas, geralmente mais frágeis diante das mudanças (MANOEL, ALMEIDA \& VILELA, 2014). Na tentativa de subsidiar estratégias de conservação e recuperação ambiental é necessário ampliar o conhecimento taxonômico da ictiofauna e compreender a sua relação com o ambiente (FERREIRA \& CASSATTI, 2006).

Neste sentido, o objetivo deste trabalho foi avaliar a estrutura e composição da ictiofauna do córrego Moeda, afluente da margem direita do rio Paraná, município de Três Lagoas, MS.

\section{MATERIAL E MÉTODOS}


Foram realizadas oito coletas trimestrais entre fevereiro/2012 e novembro/2013, em duas áreas amostrais: P1, a montante da ponte sobre a rodovia MS-395 (20 $56^{\prime} 28,7^{\prime \prime}$ S e 51 $\left.41^{\circ} 48,8^{\prime \prime} \mathrm{W}\right)$, e P2, na foz do córrego Moeda $\left(20^{\circ} 00^{\prime} 45,5^{\prime \prime}\right.$ S e $\left.51^{\circ} 45^{\prime} 28,4^{\prime \prime} \mathrm{W}\right)$, no município de Três Lagoas, Mato Grosso do Sul (Figura 1).

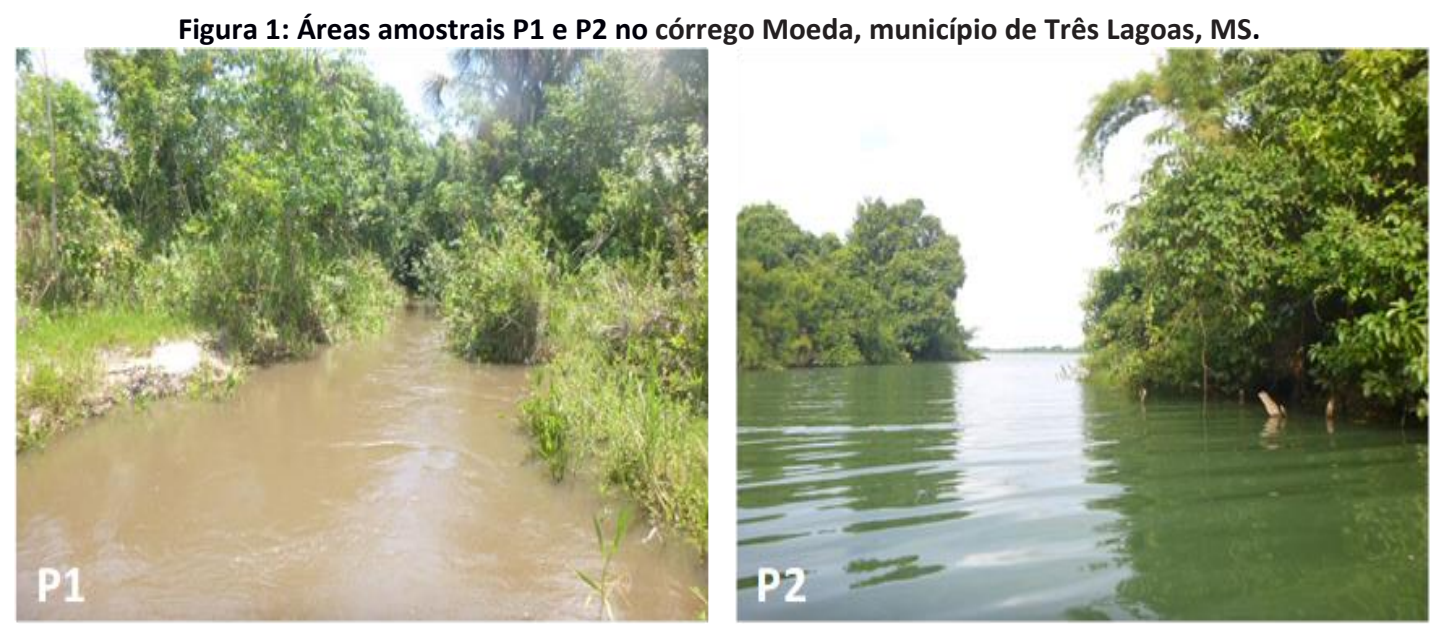

Fonte: Próprio autor.

$\mathrm{Na}$ amostragem de ictiofauna foram utilizados os seguintes petrechos de pesca experimental: a) Rede de arrasto com $10 \mathrm{~m}$ de comprimento e malha de $2,5 \mathrm{~mm}$; b) Redes de emalhar: em cada estação amostral utilizou-se uma bateria de redes de espera, contendo quatro redes de 10 metros de comprimento e 1,5 metros de altura, com malhas de 3, 6, 10 e $16 \mathrm{~cm}$ medidos entre nós opostos, e c) Peneira quadrada de $1 \times 1 \mathrm{~m}$ e malha de $2,5 \mathrm{~mm}$ (Figura 2).

Figura 2: Métodos de coleta de peixes realizados no córrego Moeda, município de Três Lagoas, MS. (A) rede de espera, $(B)$ rede de arrasto e $(C)$ peneira

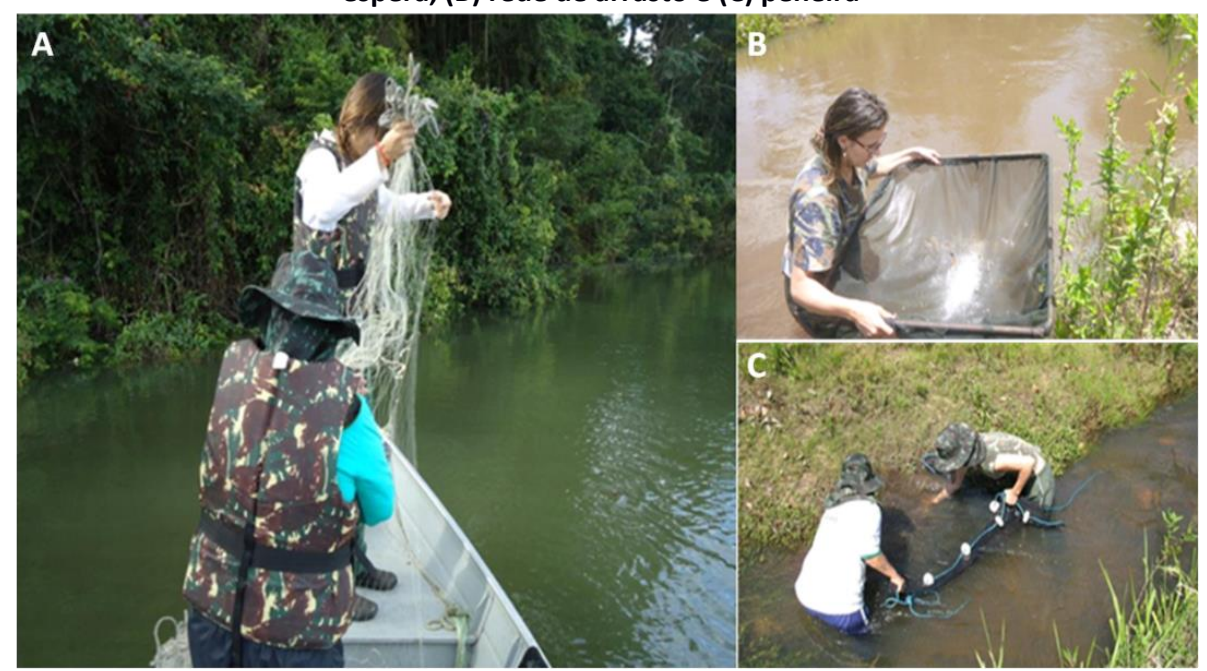

Fonte: Próprio autor.

Em campo foram realizadas a triagem das amostras e a identificação dos exemplares, procedendo-se então à biometria, que consistiu na medição do comprimento padrão $(\mathrm{cm})$ e da 
massa corporal (g). Os exemplares capturados e identificados no local foram manuseados em tempo necessário para sua devolução ao ambiente. Os peixes que não apresentavam condições de sobrevivência foram acondicionados em sacos plásticos etiquetados, separados por estação, tipo de ambiente e artefato de pesca e fixados em formalina $10 \%$.

Em laboratório, os exemplares foram transferidos para solução de álcool 70\% e disponibilizados para depósito como material testemunho no Laboratório de Ictiologia da Universidade Federal de Mato Grosso do Sul - Campus Três Lagoas.

Os peixes capturados foram identificados com base nos trabalhos de Graça e Pavanelli (2007) e Langeani et al. (2007), consultando-se também Langeani (1989), Britski et al. (1999) e Ota et al. (2018). A classificação quanto à origem e causa de ocorrência na bacia do alto Paraná segue Langeani et al. (2007).

Foram calculados riqueza de espécies (S), abundância numérica, índice de diversidade de Shannon-Wienner $\left(\mathrm{H}^{\prime}\right)$ e equitabilidade de Pielou (J) (Krebs, 1989) para cada sítio amostral.

A abundância numérica relativa de cada espécie, o índice de diversidade de Shannon-Wienner $\left(H^{\prime}\right)$ e riqueza de espécies (S) (Krebs, 1989), para ambas as áreas, foram submetidas à análise de normalidade e comparados com teste estatístico pertinente, sendo o teste-t paramétrico para a abundância numérica relativa e riqueza de espécies (S), o Teste-U Mann-Whitney para diversidade de Shannon-Wienner $\left(\mathrm{H}^{\prime}\right)$ e Teste T-Independente para equitabilidade de Pielou.

Para verificar diferenças na estrutura da comunidade entre as áreas aplicou-se a análise PERMANOVA one-way (Anderson, 2001) e posteriormente análise SIMPER, para verificar a contribuição de cada espécie para diferença observada, ambas utilizando a distância de BrayCurtis (CLARKE, 1993). Todas as análises estatísticas foram realizadas com auxílio dos softwares Past 3.0 e BioEstat 5.0 e o nível de significância adotado foi $p<0,05$.

\section{RESULTADOS E DISCUSSÃO}

Foram capturados 296 exemplares (P1 = 127 e P2 = 169), distribuídos em cinco ordens, 17 famílias, 33 gêneros e 41 espécies (Tabela 1). 
Tabela 1: Lista das espécies, abundância numérica, origem e causa de ocorrência dos peixes coletados no córrego Moeda, afluente da margem direita do rio Paraná, município de Três Lagoas, MS.

\begin{tabular}{|c|c|c|c|c|}
\hline \multirow[b]{2}{*}{ Espécies } & \multicolumn{2}{|c|}{ Abundância numérica } & \multirow[b]{2}{*}{ Origem } & \multirow[b]{2}{*}{ Ocorrência } \\
\hline & $\begin{array}{c}\text { Médio } \\
\text { curso }\end{array}$ & Foz & & \\
\hline \multicolumn{5}{|l|}{ ORDEM CHARACIFORMES } \\
\hline \multicolumn{5}{|l|}{ Família Crenuchidae } \\
\hline Characidium aff. zebra Eigenmann, 1909 & 3 & 0 & autóctone & nativa \\
\hline \multicolumn{5}{|l|}{ Família Parodontidae } \\
\hline Steindachnerina insculpta (Fernández-Yépez, 1948) & 0 & 1 & autóctone & nativa \\
\hline \multicolumn{5}{|l|}{ Família Prochilodontidae } \\
\hline Prochilodus lineatus (Valenciennes, 1836) & 0 & 1 & autóctone & nativa \\
\hline \multicolumn{5}{|l|}{ Família Anostomidae } \\
\hline Leporinus friderici (Bloch, 1794) & 0 & 2 & autóctone & nativa \\
\hline
\end{tabular}

Tabela 1: Lista das espécies, abundância numérica, origem e causa de ocorrência dos peixes coletados no córrego Moeda, afluente da margem direita do rio Paraná, município de Três Lagoas, MS (Continuação).

\begin{tabular}{|c|c|c|c|c|}
\hline Leporinus octofasciatus Steindachner, 1915 & 0 & 1 & autóctone & nativa \\
\hline Schizodon borellii (Boulenger, 1900) & 2 & 18 & autóctone & nativa \\
\hline Schizodon nasutus Kner, 1858 & 0 & 2 & autóctone & nativa \\
\hline \multicolumn{5}{|l|}{ Família Characidae } \\
\hline Aphyocharax anisitsi Eigenmann \& Kennedy, 1903 & 0 & 5 & alóctone & Itaipu \\
\hline Astyanax fasciatus (Cuvier, 1819) & 3 & 0 & autóctone & nativa \\
\hline Astyanax lacustris (Lütken, 1875) & 10 & 29 & autóctone & nativa \\
\hline Knodus moenkhausii (Eigenmann \& Kennedy, 1903) & 56 & 8 & alóctone & Itaipu \\
\hline $\begin{array}{l}\text { Moenkhausia aff. sanctaefilomenae (Steindachner, } \\
\text { 1907) }\end{array}$ & 11 & 4 & autóctone & nativa \\
\hline Moenkhausia intermedia Eigenmann, 1908 & 5 & 0 & autóctone & nativa \\
\hline Serrasalmus maculatus Kner, 1858 & 0 & 2 & autóctone & nativa \\
\hline Serrasalmus marginatus Valenciennes, 1837 & 2 & 7 & autóctone & nativa \\
\hline Hyphessobrycon eques (Steindachner, 1882) & 0 & 2 & autóctone & nativa \\
\hline Metynnis lippincottiannus (Cope, 1870) & 0 & 5 & alóctone & Itaipu \\
\hline Serrapinnus heterodon (Eigenmann, 1915) & 2 & 0 & autóctone & nativa \\
\hline Serrapinnus notomelas (Eigenmann, 1915) & 5 & 1 & autóctone & nativa \\
\hline \multicolumn{5}{|l|}{ Família Lebiasinidae } \\
\hline Pyrrhulina australis Eigenmann \& Kennedy, 1903 & 7 & 0 & autóctone & nativa \\
\hline \multicolumn{5}{|l|}{ Família Acestrorhynchidae } \\
\hline Acestrorhynchus lacustris (Lütken, 1875) & 0 & 17 & autóctone & nativa \\
\hline \multicolumn{5}{|l|}{ Família Cynodontidae } \\
\hline Rhaphiodon vulpinus Spix \& Agassiz, 1829 & 0 & 6 & autóctone & nativa \\
\hline \multicolumn{5}{|l|}{ Família Erythrinidae } \\
\hline Hoplias aff. malabaricus (Bloch, 1794) & 1 & 4 & autóctone & nativa \\
\hline \multicolumn{5}{|l|}{ Família Hemiodontidae } \\
\hline Hemiodus orthonops Eigenmann \& Kennedy, 1903 & 0 & 4 & alóctone & Itaipu \\
\hline \multicolumn{5}{|l|}{ ORDEM SILURIFORMES } \\
\hline \multicolumn{5}{|l|}{ Família Loricariidae } \\
\hline Hisonotus francirochai (Ihering, 1928) & 4 & 0 & autóctone & nativa \\
\hline Hypostomus margaritifer (Regan, 1908) & 2 & 4 & autóctone & nativa \\
\hline $\begin{array}{l}\text { Pterygoplichthys anisitsi Eigenmann \& Kennedy, } \\
1903\end{array}$ & 2 & 2 & autóctone & nativa \\
\hline Rhinelepis aspera Spix \& Agassiz, 1829 & 5 & 4 & autóctone & nativa \\
\hline \multicolumn{5}{|l|}{ Família Heptapteridae } \\
\hline Imparfinis schubarti (Gomes, 1956) & 2 & 0 & autóctone & nativa \\
\hline \multicolumn{5}{|l|}{ Família Pimelodidae } \\
\hline Pimelodus ornatus Kner, 1858 & 0 & 1 & alóctone & Itaipu \\
\hline Pimelodus maculatus La Cepède, 1803 & 1 & 10 & autóctone & nativa \\
\hline Sorubim lima (Bloch \& Schneider, 1801) & 1 & 3 & alóctone & desconhecida \\
\hline \multicolumn{5}{|l|}{ Família Doradidae } \\
\hline Pterodoras granulosus (Valenciennes, 1821) & 0 & 5 & alóctone & Itaipu \\
\hline
\end{tabular}




\begin{tabular}{|c|c|c|c|c|}
\hline $\begin{array}{l}\text { Trachydoras paraguayensis (Eigenmann \& Ward, } \\
\text { 1907) }\end{array}$ & 0 & 3 & alóctone & Itaipu \\
\hline \multicolumn{5}{|l|}{ Família Auchenipteridae } \\
\hline Parauchenipterus galeatus (Linnaeus, 1766) & 0 & 7 & autóctone & nativa \\
\hline \multicolumn{5}{|l|}{ ORDEM CYPRINODONTIFORMES } \\
\hline \multicolumn{5}{|l|}{ Família Rivulidae } \\
\hline Melanorivulus apiamici (Costa, 1989) & 1 & 0 & autóctone & nativa \\
\hline \multicolumn{5}{|l|}{ ORDEM CICHLIFORMES } \\
\hline \multicolumn{5}{|l|}{ Família Cichlidae } \\
\hline Cichla kelberi Kullander \& Ferreira, 2006 & 0 & 5 & alóctone & pesca \\
\hline Geophagus sveni Lucinda, Lucena \& Assis, 2010 & 1 & 3 & alóctone & $\begin{array}{l}\text { piscicultura/aquaris } \\
\text { mo }\end{array}$ \\
\hline Satanoperca pappaterra (Heckel, 1840) & 0 & 2 & alóctone & piscicultura \\
\hline
\end{tabular}

Tabela 1: Lista das espécies, abundância numérica, origem e causa de ocorrência dos peixes coletados no córrego Moeda, afluente da margem direita do rio Paraná, município de Três Lagoas, MS (Continuação).

\begin{tabular}{lllll}
\hline Crenicichla britskii Kullander, 1982 & 1 & 0 & autóctone & nativa \\
ORDEM PLEURONECTIFORMES & & & & \\
$\begin{array}{l}\text { Família Achiridae } \\
\text { Catathyridium jenynsii (Günther, 1862) }\end{array}$ & 0 & 1 & alóctone & Itaipu \\
\hline
\end{tabular}

Em termos de abundância numérica, em ambas as áreas a ordem Characiformes foi a mais abundante, com destaque para Knodus moenkhausii e Astyanax lacustris, seguida por Siluriformes, destacando-se Pimelodus maculatus, principalmente no sítio P2. Observou-se baixa abundância para Cichliformes, Pleuronectiformes e Cyprinodontiformes (Figura 3A). Quanto à riqueza de espécies, os maiores valores foram de Characiformes e Siluriformes, seguidas de Cichliformes, corroborando com o padrão esperado para ambientes de água doce neotropical (LOWE-MCCONNELL, 1999) (Figura 3B). 
Figura 3: Participação das ordens de peixes em número de indivíduos (A) e riqueza de espécies (B) nos sítios amostrais no córrego Moeda, município de Três Lagoas, MS.
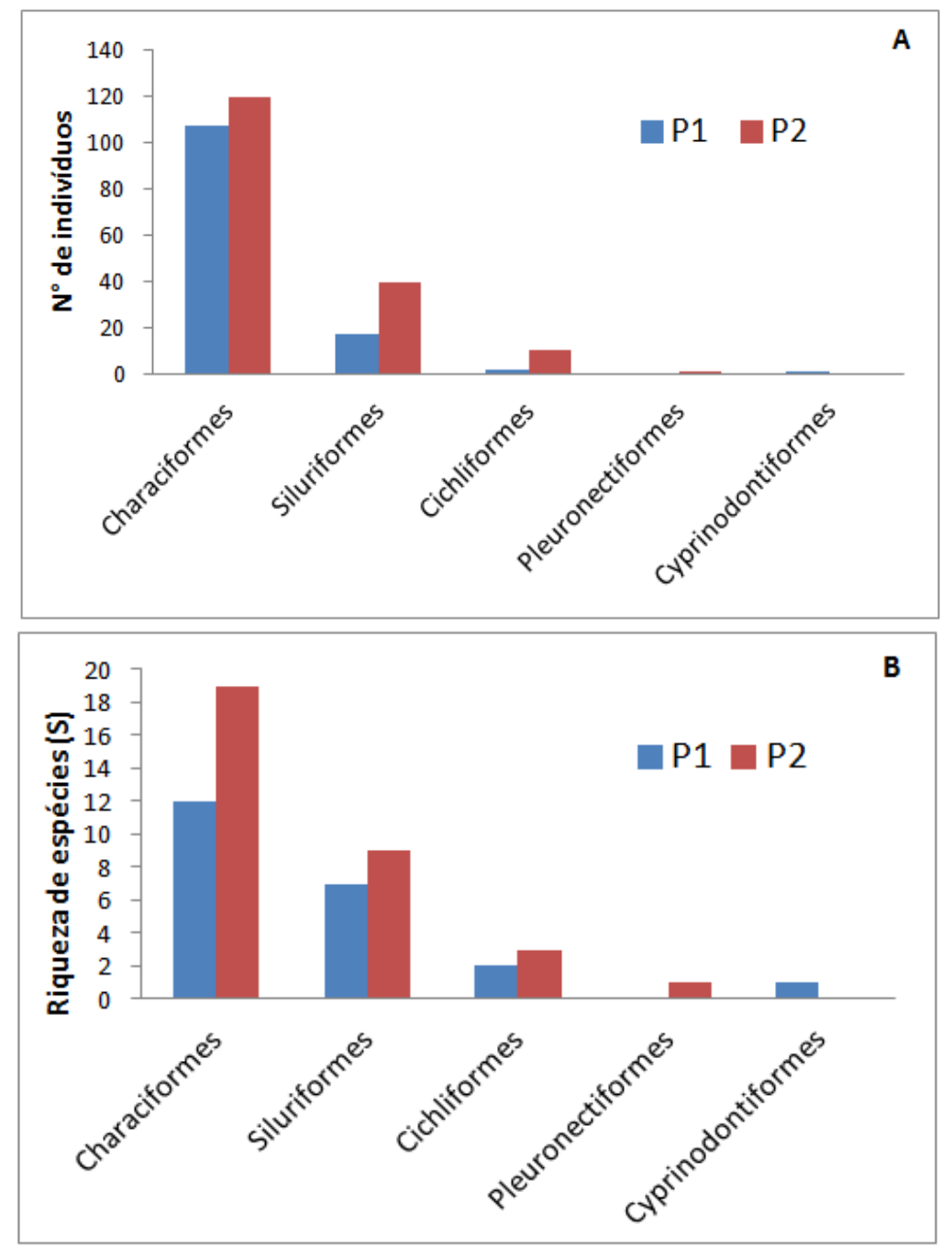

Fonte: Próprio autor

Em relação à origem da fauna, no $\mathrm{P} 1$ foram registradas 19 espécies autóctones (aquelas que ocorrem naturalmente no alto rio Paraná) e três introduzidas (alóctones). No P2, 20 espécies eram autóctones e 12 alóctones (Figura 4). Conforme Langeani et al. (2007), entre as introduzidas, Aphyocharax anisitsi, Knodus moenkhausii, Metynnis lippincottianus, Hemiodus orthonops, Pimelodus ornatus, Pterodoras granulosus, Trachydoras paraguayensis e Catathyridium jenynsii são espécies que dispersaram-se pelo alto rio Paraná após a construção do Reservatório de Itaipu; Satanoperca pappaterra, introduzida intencional ou acidentalmente e amplamente utilizada em pisciculturas; Geophagus sveni, uma espécie de aquário introduzida intencional ou acidentalmente no rio Paraná; Cichla kelberi, introduzida para a pesca esportiva ou para uso como iscas, e Sorubim lima, cuja causa de ocorrência é desconhecida. 
Figura 4: Participação das espécies de peixes quanto à origem da fauna nos sítios amostrais no córrego Moeda, município de Três Lagoas, MS.

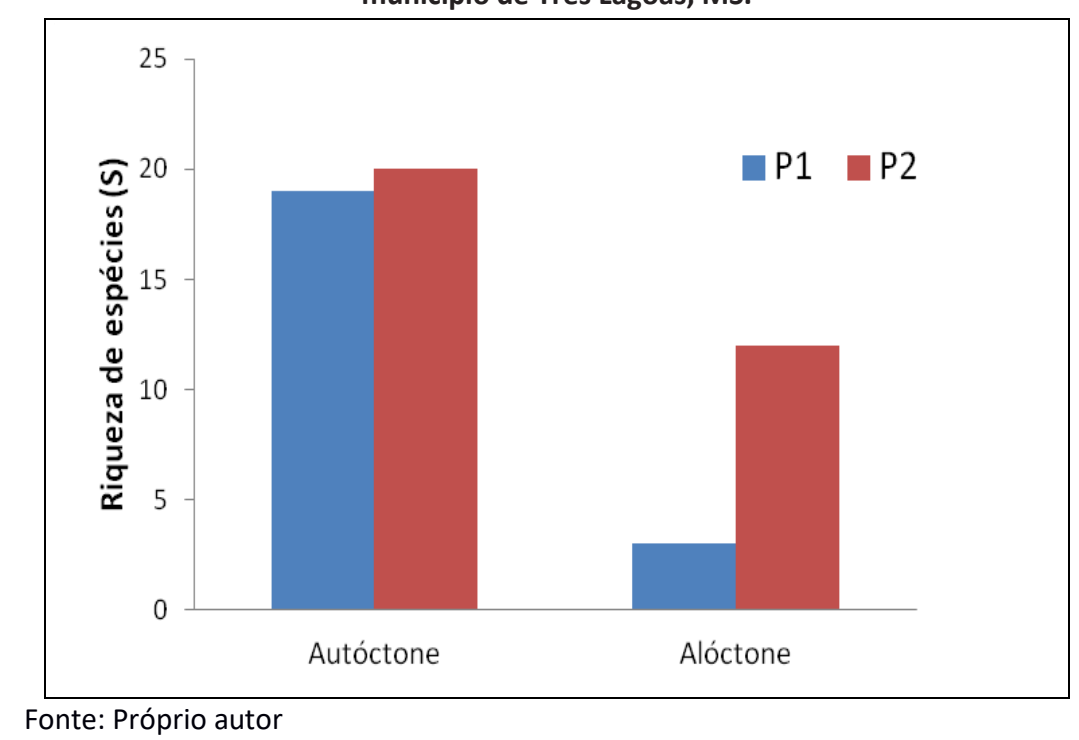

Nas análises dos atributos da comunidade não se observou diferença quanto à abundância numérica $(P 1=127$ e $P 2=169, p=0.235)$ e à equitabilidade entre as áreas avaliadas $(P 1=0.718$ e $P 2=0.876, p=0,914)$. Contudo, houve diferença quanto à riqueza de espécies ( $P 1=22$ e $P 2=32$, $p=0,004)$ e à diversidade de Shannon-Wiener $(P 1=2,222$ e $P 2=3,037, p=0,024)$, conforme Tabela 2.

Tabela 2. Atributos ecológicos da ictiofauna para os sítios amostrais no córrego Moeda, município de Três Lagoas, MS.

\begin{tabular}{|c|c|c|c|}
\hline \multirow[b]{2}{*}{ Atributos } & \multicolumn{2}{|c|}{ Estações de amostragem } & \multirow[b]{2}{*}{$\mathbf{P}$} \\
\hline & $\begin{array}{l}\text { Montante } \\
\text { (P1) }\end{array}$ & Jusante (P2) & \\
\hline Abundância & 127 & 169 & $p=0.235$ \\
\hline Riqueza (S) & 22 & 32 & $p=0.004 *$ \\
\hline Diversidade $\left(\mathrm{H}^{\prime}\right)$ & 2.222 & 3.037 & $p=0.024^{*}$ \\
\hline Equitabilidade (J) & 0.7188 & 0.8764 & $p=0.914$ \\
\hline
\end{tabular}

Observaram-se diferenças na estrutura da comunidade (PERMANOVA $\mathrm{p}=0,0005$ ), sendo Knodus moenkhausii $(16,7 \%)$ e Astyanax lacustris $(9,8 \%)$ as espécies que mais contribuíram para a diferença, segundo análise SIMPER (Tabela 3). Cabe destacar que essas espécies possuem grande plasticidade fenotípica e são tolerantes às interferências antrópicas (BENEDITO-CECÍLIO e AGOSTINHO, 1997; CENEVIVA-BASTOS \& CASATTI, 2007). 
Tabela 3. Análise de SIMPER para as espécies de peixes coletadas nos sítios amostrais no córrego Moeda, município de Três Lagoas, MS.

\begin{tabular}{|c|c|c|c|c|}
\hline Espécies & Contribuição & Acumulado \% & P1 & P2 \\
\hline Knodus moenkhausii & 16.66 & 19.04 & 7 & 1 \\
\hline Astyanax lacustris & 9.777 & 30.21 & 1.25 & 3.63 \\
\hline Schizodon borellii & 5.289 & 36.26 & 0.25 & 2.25 \\
\hline Acestrorhynchus lacustris & 4.671 & 41.6 & 0 & 2.13 \\
\hline Moenkhausia aff. sanctaefilomenae & 4.077 & 46.26 & 1.38 & 0.5 \\
\hline Pimelodus maculatus & 3.853 & 50.66 & 0.125 & 1.25 \\
\hline Pyrrhulina australis & 2.818 & 53.88 & 0.875 & 0 \\
\hline Rhinelepis aspera & 2.809 & 57.09 & 0.625 & 0.5 \\
\hline Serrasalmus marginatus & 2.568 & 60.02 & 0.25 & 0.875 \\
\hline Aphyocharax anisitsi & 2.291 & 62.64 & 0 & 0.625 \\
\hline Parauchenipterus galeatus & 2.149 & 65.1 & 0 & 0.875 \\
\hline Serrapinnus notomelas & 2.106 & 67.5 & 0.625 & 0.125 \\
\hline Hypostomus margaritifer & 2.037 & 69.83 & 0.25 & 0.5 \\
\hline Metynnis lippincottianus & 2.037 & 72.16 & 0 & 0.625 \\
\hline Rhaphiodon vulpinus & 1.99 & 74.43 & 0 & 0.75 \\
\hline Pterodoras granulosus & 1.896 & 76.6 & 0 & 0.625 \\
\hline Hoplias aff. malabaricus & 1.731 & 78.58 & 0.125 & 0.5 \\
\hline Cichla kelberi & 1.639 & 80.45 & 0 & 0.625 \\
\hline Hisonotus francirochai & 1.462 & 82.12 & 0.5 & 0 \\
\hline Hemiodus orthonops & 1.334 & 83.65 & 0 & 0.5 \\
\hline Sorubim lima & 1.299 & 85.13 & 0.125 & 0.375 \\
\hline Pterygoplichthys anisitsi & 1.267 & 86.58 & 0.25 & 0.25 \\
\hline Moenkhausia intermedia & 1.26 & 88.02 & 0.625 & 0 \\
\hline Geophagus sveni & 1.072 & 89.24 & 0.125 & 0.375 \\
\hline Serrapinnus heterodon & 0.9066 & 90.28 & 0.25 & 0 \\
\hline Trachydoras paraguayensis & 0.8851 & 91.29 & 0 & 0.375 \\
\hline Characidium aff. zebra & 0.8727 & 92.29 & 0.375 & 0 \\
\hline Astyanax fasciatus & 0.7562 & 93.15 & 0.375 & 0 \\
\hline Schizodon nasutus & 0.7492 & 94.01 & 0 & 0.25 \\
\hline Imparfinis schubarti & 0.7134 & 94.82 & 0.25 & 0 \\
\hline Serrasalmus maculatus & 0.6935 & 95.62 & 0 & 0.25 \\
\hline
\end{tabular}


Tabela 3. Análise de SIMPER para as espécies de peixes coletadas nos sítios amostrais no córrego Moeda, município de Três Lagoas, MS (Continuação).

\begin{tabular}{lcccc}
\hline Hyphessobrycon eques & 0.6253 & 96.33 & 0 & 0.25 \\
Leporinus friderici & 0.5523 & 96.96 & 0 & 0.25 \\
Satanoperca pappaterra & 0.4792 & 97.51 & 0 & 0.25 \\
Prochilodus lineatus & 0.3809 & 97.95 & 0 & 0.125 \\
Steindachnerina insculpta & 0.3809 & 98.38 & 0 & 0.125 \\
Crenicichla britskii & 0.3678 & 98.8 & 0.125 & 0 \\
Melanorivulus apiamici & 0.3103 & 99.16 & 0.125 & 0 \\
Pimelodus ornatus & 0.2598 & 99.45 & 0 & 0.125 \\
Catathyridium jenynsii & 0.2396 & 99.73 & 0 & 0.125 \\
Leporinus octofasciatus & 0.2396 & 100 & 0 & 0.125 \\
\hline
\end{tabular}

Quanto às análises da estrutura da comunidade, os resultados demonstraram diferenças na riqueza e diversidade de espécies entre os sítios amostrais. Tal resultado pode estar relacionado à maior heterogeneidade de habitat em $\mathrm{P} 2$, como mata ciliar mais preservada, presença de macrófitas aquáticas e de troncos submersos, que oferecem locais para forrageamento, reprodução e abrigo contra predadores, além de maior proximidade à calha do rio Paraná. No sítio $\mathrm{P} 1$, ao contrário, a mata ripária encontra-se bastante reduzida, ausente em alguns trechos, refletindo em maior assoreamento, menor profundidade e menor variabilidade do substrato, aspectos que devem colaborar para a diminuição da diversidade de peixes, o que pode justificar, pelo menos em parte, os resultados observados.

Segundo o conceito de contínuo fluvial é esperado um aumento gradual na riqueza de espécies ao longo do gradiente longitudinal. Na região tropical, estudos feitos em alguns riachos relativamente íntegros demonstraram a adição de espécies no sentido montante-jusante, acompanhando o incremento da complexidade física e estrutural dos ambientes (VANNOTE et al. 1980).

Segundo Ferreira \& Casatti (2006), com a supressão da vegetação ripária e consequente aumento da incidência de luz no substrato, a produtividade primária autóctone pode ser incrementada. Contudo, ao substituir a base alóctone pela autóctone como fonte energética para a biota aquática, é provável que determinadas espécies sejam excluídas e outras favorecidas.

\section{CONCLUSÃO}

Os resultados apontaram influência da estrutura física de cada trecho sobre a estrutura das ictiocenoses, sendo que as maiores diversidades específicas foram registradas nos trechos de maior complexidade estrutural, confirmando a importância da preservação da vegetação marginal dos riachos, essencial à manutenção da biota aquática. 


\section{REFERÊNCIAS BIBLIOGRÁFICAS}

AGOSTINHO, Angelo Antônio et al. Migratory fishes of the upper Paraná River basin. In Migratory fishes of South America: biology, fisheries and conservation status. World Fisheries Trust, World Bank, IDRC, 2004.

AGOSTINHO, Angelo Antônio; GOMES, Luiz Carlos. O manejo da pesca em reservatórios da bacia do Alto rio Paraná: avaliação e perspectivas. In: NOGUEIRA, M.G.; HENRY, R.; JORCIN, A. (Orgs.). Ecologia de reservatórios: impactos potenciais, ações de manejo e sistemas em cascata. São Carlos: RiMa. 2006, p23-55.

ANDERSON, Marti Jane. A new method for non-parametric multivariate analysis of variance. Austral Ecology, v.26, p.32-46, 2001.

BENEDITO-CECILIO, Evanilde; AGOSTINHO, Angelo Antônio. Estrutura das populações de peixes no reservatório de Segredo. In Reservatório de Segredo: bases ecológicas para o manejo (A.A. Agostinho \& L.C. Gomes, orgs.). EDUEM, Maringá, p. 113-139, 1997.

BRITSKI, Heraldo A. et al. Peixes do Pantanal: Manual de Identificação. Brasília, DF, EMBRAPA-SPI, 1999, p.184.

BUCKUP, Paulo Andreas et al. Catálogo das espécies de peixes de água doce do Brasil. Rio de Janeiro: Museu Nacional. 2007, 195p.

CASATTI, Lilian et al. Fish community structure along a conservation gradient in Bodoquena Plateau streams, central West of Brazil. Acta Limnologica Brasiliensia, 50-59, 2010.

CASATTI, Lilian; FERREIRA, Cristiane P.; LANGEANI, Francisco. A fish-based biotic integrity index for assessment of lowland streams in southeastern Brazil. Hydrobiologia, v. 623, n. 1, p. 173-189, 2009.

CENEVIVA-BASTOS, Mônica; CASATTI, Lilian. Oportunismo alimentar de Knodus moenkhausii (Teleostei, Characidae): uma espécie abundante em riachos do noroeste do Estado de São Paulo, Brasil. Iheringia: Série Zoologia, p. 7-15, 2007.

CLARKE, K. Robert. Non-parametric multivariate analysis of changes in community structure. Australian Journal of Ecology, v.18, p.117-143, 1993.

FERREIRA, Cristiane de P.; CASATTI, Lilian. Influência da estrutura do habitat sobre a ictiofauna de um riacho em uma micro-bacia de pastagem, São Paulo Brasil. Revista Brasileira de Zoologia, v.23, n.3, p. 642-651. 2006.

DA GRACA, Weferson Júnio et al. Peixes da planície de inundação do Alto rio Paraná e áreas adjacentes. Maringá: EDUEM, 2007.

KREBS, Charles J. Ecological methodology. New York: Collins, 1989.

LANGEANI NETO, Francisco. Ictiofauna do Alto curso do Rio Tietê (SP): Taxonomia. Dissertação de Mestrado, Instituto de Biociências da Universidade de São Paulo. 1989.

LANGEANI NETO, Francisco et al. Diversidade da ictiofauna do Alto Rio Paraná: composição atual e perspectivas futuras. Biota Neotropica, p. 181-197, 2007.

LANGEANI, Francisco et al. Diversidade da ictiofauna do Alto Rio Paraná: composição atual e perspectivas futuras. Biota Neotropica. 2007.

LORION, Christopher M.; KENNEDY, Brian P. Riparian forest buffers mitigate the effects of deforestation on fish assemblages in tropical headwater streams. Journal of Applied Ecology, v. 19, n. 2, p. 468-479, 2009.

LOWE-MCCONNELL, Rosemary H. Estudos ecológicos em comunidades de peixes tropicais. Editora da Universidade de São Paulo, São Paulo, 1999, 534p. 
MANOEL, Letícia de Oliveira et al. Composição da ictiofauna do córrego Santa Vera, alto rio Paraná, Três Lagoas, MS (2012-2014). Periódico Eletrônico Fórum Ambiental da Alta Paulista, v. 10, n. 2, 2014.

OTA, Renata Rúbia et al. Peixes da planície de inundação do alto rio Paraná e áreas adjacentes: revised, annotated and updated. Neotropical Ichthyology, v. 16, n. 2, 2018.

VANNOTE, Robin L. et al. The river continuum concept. Canadian Journal of Fisheries and Aquatic Sciences, Ottawa, 37: 130-137. 1980.

VARI, Richard. P; MALABARBA, Luiz R. Neotropical ichthyology: an overview. In Phylogeny and classification of neotropical fishes (L.R. Malabarba, R.E. Reis, R.P. Vari \& Z.M.S. Lucena, eds.). Edipucrs, Porto Alegre, p. 1-11, 1998. 